\title{
Identification of mutations in SLC4A1, GP1BA and HFE in a family with venous thrombosis of unknown cause by next-generation sequencing
}

\author{
WEI-AN CHANG ${ }^{1,2}$, CHAU-CHYUN SHEU ${ }^{1-3}$, KUAN-TING LIU $^{1,3,4}$, \\ JHENG-HENG SHEN ${ }^{1,4}$, MENG-CHI YEN ${ }^{1,4}$ and PO-LIN KUO ${ }^{1}$ \\ ${ }^{1}$ Graduate Institute of Clinical Medicine, College of Medicine, Kaohsiung Medical University; \\ ${ }^{2}$ Division of Pulmonary and Critical Care Medicine, Kaohsiung Medical University Hospital; \\ ${ }^{3}$ School of Medicine, College of Medicine, Kaohsiung Medical University; ${ }^{4}$ Department of Emergency Medicine, \\ Kaohsiung Medical University Hospital, Kaohsiung Medical University, Kaohsiung 807, Taiwan, R.O.C.
}

Received February 12, 2018; Accepted May 17, 2018

DOI: $10.3892 /$ etm.2018.6693

\begin{abstract}
Various risk factors, including high age, female gender, obesity and certain genetic defects have been linked to venous thrombosis. A Taiwanese family with venous thrombosis of unknown cause were enrolled in the present study. In this pedigree, two women without any specific underlying diseases suffered from venous thrombotic events at the same age. No specific risk factors or coagulation abnormalities were identified. The main proband's younger brother also had intestinal arterial thrombosis at 54 years of age. Therefore, it was hypothesized that familial genetic defects may be the cause of venous thrombosis within this family. Blood samples collected from certain members of this pedigree were subjected to whole-exome sequencing, and three genetic variants were identified, including a missense variant of solute carrier family 4 member 1 (SLC4A1) (c.388G>A), a deletion on glycoprotein Ib platelet $\alpha$ subunit (GP1BA) (c.1322_1344del23) and an insertion in the splice site of homeostatic iron regulator (HFE). To date, none of these three genetic variants have been reported to be associated with venous thrombosis, to the best of our knowledge. The present study suggests that these genetic variants of SLC4A1, GP1BA and HFE may be associated with venous thrombosis in an Asian pedigree.
\end{abstract}

Correspondence to: Dr Meng-Chi Yen, Department of Emergency Medicine, Kaohsiung Medical University Hospital, Kaohsiung Medical University, 100 Shih-Chuan 1st Road, Kaohsiung 807, Taiwan, R.O.C.

E-mail: yohoco@gmail.com

Key words: venous thrombosis, next-generation sequencing, whole-exome sequencing, solute carrier family 4 member 1 , glycoprotein Ib platelet $\alpha$ subunit, homeostatic iron regulator

\section{Introduction}

Venous thrombosis is a common vascular event where a blood clot forms within a vein. Age, surgery, obesity, trauma, pregnancy and hormone replacement therapy are known risk factors of venous thrombosis. Deficiencies of anti-thrombin, protein $\mathrm{C}$ and protein $\mathrm{S}$ also pre-dispose to thrombosis. In addition, genetic defects in fibrinogen, prothrombin, factor $\mathrm{V}$ Leiden, coagulation factor XIII and coagulation factor XI are considered as inherited risk factors (1-3). With the advance of sequencing technology, rare and low-frequency genetic variants may now be easily detected. Various novel genetic polymorphisms associated with venous thrombosis, including deep venous thrombosis (DVT), have been identified (4-6). Understanding of these rare inherited risk factors may facilitate risk stratification and prophylaxis in clinical practice. Venous thrombosis usually occurs in thrombosis of veins in the leg and pulmonary embolism (1); however, venous thrombosis may also occur in mesenteric veins and cause mesenteric venous thrombosis. Primary mesenteric venous thrombosis is usually spontaneous or idiopathic (7), accounting for $10-15 \%$ of all mesenteric ischemic events. Mesenteric ischemia should be diagnosed and treated promptly, as the mortality rate may reach $70 \%$ if the time to diagnosis is $>24 \mathrm{~h} \mathrm{(8)}$.

The present study presents the case of a 64-year-old, previously healthy Taiwanese woman admitted to Kaohsiung Medical University Hospital (KMUH; Kaohsiung, Taiwan) with mesenteric ischemia. The older sister of the proband also suffered from DVT of the femoral vein at the same age. The younger brother of the proband had intestinal arterial thrombosis when he was 54 years of age. None of the patients had any specific underlying diseases or risk factors for venous thrombosis; therefore, it was hypothesized that familial genetic defects may pre-dispose this pedigree to venous thrombosis. Peripheral blood samples were collected from the proband, the proband's daughter and the proband's older sister for next-generation sequencing (NGS). Combined with bioinformatics, the present study aimed to identify genetic factors associated with venous thrombosis in this pedigree. 


\section{Materials and methods}

Study subjects. A female patient (age, 64 years) was admitted to KMUH (Kaohsiung, Taiwan) with mesenteric venous thrombosis of unknown cause. The proband's older sister (thrombosis occurrence at 64 years; current age, 66 years) and younger brother (thrombosis occurrence at 54 years; current age, 61 years) had also experienced venous thrombotic events of unknown cause. The surveys for risk factors of their thrombosis were all negative. In order to investigate the possible cause of venous thrombosis in this pedigree, peripheral blood samples of the proband, the proband's older sister and the proband's daughter, a healthy 32-year-old woman without any history of thrombotic events, were collected for next-generation sequencing analysis. The present study was approved by the Institutional Review Board of KMUH (Kaohsiung, Taiwan). All subjects provided written informed consent.

Library preparation and sequencing. For the generation of standard exome capture libraries, the Agilent SureSelect XT Reagent kit for the Illumina Hiseq paired-end sequencing library (cat. no. G9611 A; Agilent Technologies, Inc., Santa Clara, CA, USA) was used according to the manufacturer's protocol. In all cases, the SureSelect XT Human All Exon Version $6(60 \mathrm{Mb})$ probe set (Agilent Technologies, Inc.) was used. A total of $1 \mu \mathrm{g}$ genomic DNA (gDNA) was used to construct a library with the Agilent SureSelect XT Reagent kit. The amplification adapter-ligated sample was purified using Agencourt AMPure XP beads (Beckman Coulter, Brea, CA, USA) and analyzed on a TapeStation 4200 D1000 screen tape (Agilent Technologies, Inc.). The gDNA library (750 ng) was prepared for hybridization with the capture beads, and the sample was hybridized for $24 \mathrm{~h}$ at $65^{\circ} \mathrm{C}$, captured with the Dynabeads MyOne Streptavidin T1 (Thermo Fisher Scientific, Inc., Waltham, MA, USA) and purified using Agencourt AMPure XP beads. The Agilent protocol was used for addition of index tags (Agilent SureSelect XT Reagent kit; Illumina, Inc., San Diego, CA, USA; cat. no. G9611B) by post-hybridization amplification. Finally, all samples were sequenced on an Illumina Sequencer (Illumina, Inc., San Diego, CA, USA) using the $150 \mathrm{PE}$ protocol as specified by Illumina, Inc.

Processing of sequencing data and identification of mutations. FASTQ file quality control was performed using Trimmomatic (9). Reads from the samples were mapped to the reference sequence Human Genome version 19 (hg19) by using the Burrows-Wheeler Aligner (10). For further variant analyses, variants in coding or non-coding regions were identified, and annotation were created with a Genome Analysis Toolkit (GATK) according to the GATK best practices pipeline (11) and Variant Effect Predictor (12). These mutations were further filtered by the global minor allele frequency and ClinVar (https://www.ncbi.nlm.nih.gov/clinvar/) databases (13). The mutations identified in the ClinVar database were considered to be known variants and those not found in the ClinVar database were identified as unknown variants. Both known and unknown variants were further filtered out by using the criterion $>1 \%$ Asian minor allele frequency according to TaiwanBioBank (http://taiwanview.twbiobank.org.tw) (14).
The filtered variants were identified as known or unknown rare variants.

Functional annotation analysis. Functional annotation was performed using the Database for Annotation, Visualization and Integrated Discovery (DAVID 6.7) (https://david.ncifcrf.gov) $(15,16)$. After annotating the gene list, the potential genes were obtained through analysis of the genetic association database disease class.

Protein features and domains. The information regarding features and functional domains of protein sequences was adapted from UniProt [http://www.uniprot.org, P07359 (GP1BA_HUMAN)]. The reference sequence of GP1BA from Homo sapiens (NCBI Reference Sequence: NP_000164), Mus musculus (NCBI Reference Sequence: NP_034456) and Rattus norvegicus (NCBI Reference Sequence: NP_001103124) was obtained from the NCBI protein database (https://www.ncbi.nlm.nih.gov/protein/).

\section{Results and Discussion}

Case presentation. The case of the present study was a 64-year-old woman without any specific underlying diseases. She suffered from progressive abdominal pain for 2 weeks and was brought to the emergency department of KMUH, where abdominal computed tomography revealed venous thrombosis involving the superior mesenteric vein and splenic vein. Due to progressive abdominal pain with a significant peritoneal sign, she underwent small bowel segmentectomy and jejunostomy. After the operation, she received anti-coagulant and anti-platelet therapies. The surveys for the cause of her venous thrombosis, including examinations for cancer, auto-immune diseases and coagulation diseases, were all negative (Table I). Only elevated plasma fibrinogen and D-dimer levels were identified; however, these are common results during thrombotic events. The proband's older sister had also suffered from DVT of the left femoral vein at the age of 64 years. She did not have any specific underlying diseases or thrombosis risk factors, either. Furthermore, the proband's younger brother had intestinal arterial thrombosis at 54 years of age. Similarly, no specific underlying diseases or risk factors were identified in this patient. These rare circumstances implied that this pedigree may have familial genetic factors pre-disposing them to thrombosis.

The pedigree of the pedigree is presented in Fig. 1. The proband (II-3) had mesenteric vein thrombosis at 64 years of age. The proband's older sister (II-1) had femoral vein thrombosis at 64 years of age also. The proband's mother (I-2) had had a stroke. The proband's younger brother (II-4) had intestinal arterial thrombosis. The laboratory data of cases II-3 and II-1, as well as and the proband's daughter (III-3), were collected and compared. Most of the data were within normal ranges, except for plasma fibrinogen (Table I). The fibrinogen levels in case II-1 were slightly elevated, even though there was no active thrombotic event when the blood sample was collected. Fibrinogen is an important factor affecting platelet function (17). Furthermore, inherited fibrinogen disorders are a risk factor of thrombotic complications (18). To investigate whether any genetic variants on fibrinogen and other 
Table I. Biochemical parameters of the subjects of the present study.

\begin{tabular}{|c|c|c|c|c|}
\hline Parameters & Normal range & Proband & Proband's sister & Proband's daughter \\
\hline Age (years) & & 64 & 66 & 34 \\
\hline Gender & & Female & Female & Female \\
\hline BMI (kg/m²) & & 24.4 & 22.6 & 17.5 \\
\hline Rheumatoid factor (mg/dl) & $<15.9$ & $<10.0$ & $<10.0$ & $<10.0$ \\
\hline Lactate $(\mathrm{mmol} / \mathrm{l})$ & $0.5-2.2$ & 1.32 & $<1.2$ & $<1.2$ \\
\hline Anti-thrombin III (\%) & $75-125$ & 80 & 71 & 93 \\
\hline Protein C $(\%)$ & $70-140$ & 92 & 85 & 106 \\
\hline Protein S (\%) & $58.6-126$ & 62 & 90 & 92 \\
\hline Antinuclear antibody & $<1: 40$ & $<1: 40$ & $<1: 40$ & $<1: 40$ \\
\hline D-Dimer (mg/l) & $<0.55$ & $5.58^{\mathrm{a}}$ & $3.37^{\mathrm{a}}$ & 0.18 \\
\hline Fibrinogen (mg/dl) & $200-400$ & 387.1 & $472.0^{\mathrm{a}}$ & 198.0 \\
\hline PT (INR) & $0.85-1.15$ & 1.07 & 1.02 & 1.05 \\
\hline GLU (AC) (mg/dl) & $65-109$ & $150^{\mathrm{a}}$ & $114^{\mathrm{a}}$ & 96 \\
\hline Albumin $(\mathrm{g} / \mathrm{dl})$ & $3.5-5$ & 4.11 & 4.21 & 4.66 \\
\hline LDH (IU/l) & 98-192 & $229^{a}$ & 181 & 112 \\
\hline BUN (mg/dl) & $8-20$ & 8.6 & 9.9 & 9.6 \\
\hline Creatinine (mg/dl) & $0.44-1.03$ & 0.52 & 0.76 & 0.59 \\
\hline $\mathrm{Na}(\mathrm{mmol} / \mathrm{l})$ & $136-144$ & 140 & 136 & 141 \\
\hline $\mathrm{K}(\mathrm{mmol} / \mathrm{l})$ & $3.5-5.1$ & 3.4 & 3.7 & 3.8 \\
\hline CT (mg/dl) & $140-200$ & 98 & 164 & 174 \\
\hline TG (mg/dl) & $35-160$ & 418 & 91 & 36 \\
\hline APL IgG (GPL) & $<15$ & 7.46 & 11.21 & 4.31 \\
\hline APL IgM (MPL) & $<15$ & $<1.5$ & $>100^{\mathrm{a}}$ & 2.17 \\
\hline aCL-IgG (GPL-U/ml) & $<7$ & 10 & 1.1 & 0.6 \\
\hline aCL-IgM (MPL-U/ml) & $<10$ & 4.3 & $>472^{\mathrm{a}}$ & 2.5 \\
\hline $\mathrm{WBC}\left(\mathrm{x} 10^{3} / \mu \mathrm{l}\right)$ & $4.14-10.52$ & $11.41^{\mathrm{a}}$ & 6.98 & 5.83 \\
\hline $\mathrm{RBC}\left(\mathrm{x} 10^{6} / \mu \mathrm{l}\right)$ & $11.1-15.1$ & 3.71 & 4.06 & 4.70 \\
\hline $\operatorname{Hgb}(\mathrm{g} / \mathrm{dl})$ & $11.1-15.1$ & 10.8 & 12.6 & 13.6 \\
\hline $\operatorname{Hct}(\%)$ & $34.7-45.1$ & $33.1^{\mathrm{a}}$ & 37.3 & 42.0 \\
\hline $\operatorname{MCV}(f l)$ & $83.4-98.5$ & 89.2 & 91.9 & 89.4 \\
\hline $\operatorname{PLT}\left(\mathrm{x} 10^{3} / \mu 1\right)$ & $160-370$ & 201 & 213 & 269 \\
\hline
\end{tabular}

Indicates that the value is not within the normal range. PT (INR), prothrombin time (international normalize ratio); Glu (AC), glucose (ante cibum); LDH, lactic dehydrogenase; BUN, blood urea nitrogen; CT, total cholesterol; TG, triglyceride; APL IgG (GPL), anti-phospholipid IgG, negative; APL IgM (MPL), anti-phospholipid IgM, negative; aCL-IgG, anti-cardiolipin IgG, negative; aCL-IgM, anti-cardiolipin IgM, negative; WBC, white blood cell; RBC, red blood cell; Hgb, hemoglobin; Hct, hematocrit; MCV, mean corpuscular volume; PLT, platelets; Ig, immunoglobulin; BMI, body mass index.

unknown genes were associated with vein thrombosis within this pedigree, cases II-3, II-1 and III-3 were enrolled in the present study. Their peripheral blood samples were collected for whole-exome sequencing.

Results of whole-exome sequencing analysis: Known rare variants. After whole-exome sequencing, the results were analyzed according to the flowchart presented in Fig. 2 and the procedures specified above. Common variants were excluded from analysis. The rare variants were further verified by ClinVar annotation based on hg19. Subsequently, these genes were filtered out according to the criterion $>1 \%$ Asian minor allele frequency. Two genes with known rare variants shared between the proband and the proband's sister are presented in Table II. The first identical mutation in the proband and the proband's sister was on solute carrier family 4 member 1 (SLC4A1; GenBank ID NM_000342.3) which is a band 3 cytoplasmic domain and phosphotransferase/anion transporter (19). A heretogenous missense variant c.388G $>$ A (p.Gly130Arg, rs121912749) in exon 6 of SLC4A1 was observed. According to the ClinVar database, this mutation of SLC4A1 is pathogenic in spherocytosis type 4. Hereditary spherocytosis causes vein thrombosis in rare cases $(20,21)$. Although spherocytosis has not much in common with venous thrombosis, the Gly130Arg mutation may still lead to a decrease in anion exchange across the erythrocyte membrane, thereby affecting the shape of the erythrocytes. Such a shape change is expected to affect thrombosis. 


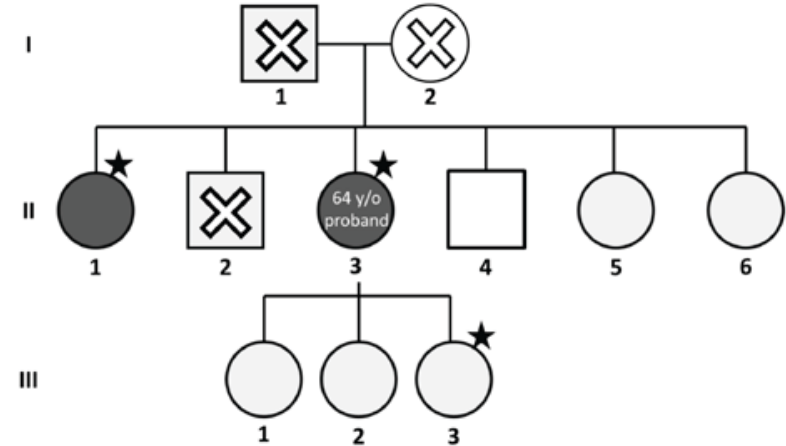

Figure 1. Pedigree chart and selection for whole-exome sequencing. Pedigree members are represented as squares (males) and circles (females). Filled and unfilled symbols indicate subjects with and without venous thrombosis, respectively. The mother (I-2) of the main proband (II-3) had a stroke. The younger brother (II-4) of the main proband had arterial thrombosis. The star symbols indicate pedigree members enrolled for next-generation sequencing in the present study. The ' $\mathrm{X}$ symbol indicates that the subject is deceased.

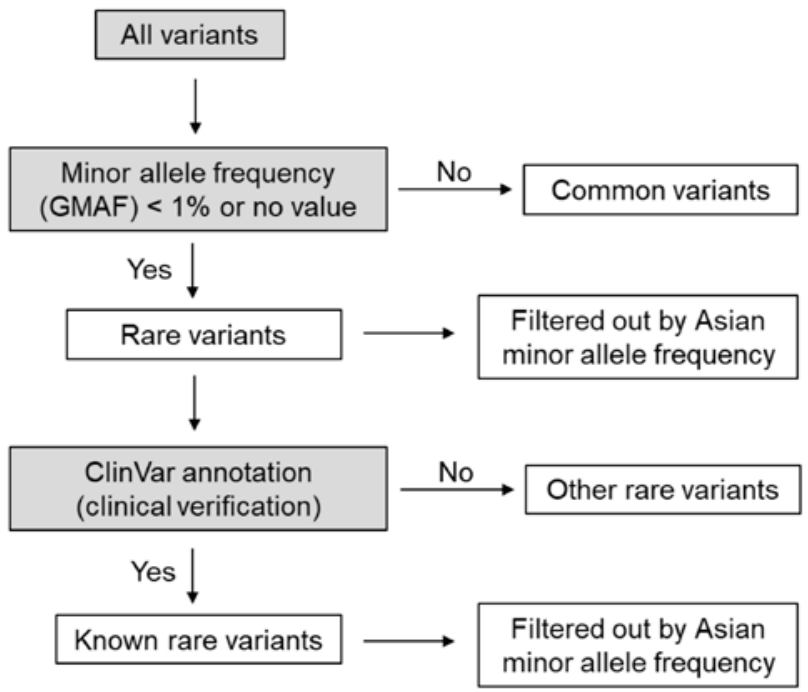

Figure 2. Flowchart depicting the analytical strategy. Steps in the analysis of the whole-exome sequencing data are presented. GMAF, global minor allele frequency.

The second gene with a rare mutation shared between the proband and the proband's sister is glycoprotein Ib platelet $\alpha$ subunit (GP1BA). GP1BA and GP1BB constitute a heterodimer of glycoprotein $\mathrm{Ib}$, which is a platelet surface membrane glycoprotein (22). In platelets, GP1B is an essential surface receptor for von Willebrand factor (VWF). The binding site of VWF is located in the leucine-rich repeat domain of GP1BA (23). The illustration of protein domains, including the leucine-rich repeat N-terminal domain [amino acids 19-46 (19-46 aa)], the NEL superfamily (34-205 aa) and the leucine-rich repeat C-terminal domain (221-281 aa) are presented in Fig. 3. Mutations within the leucine-rich repeat C-terminal domain of GP1BA (p.Asp235Tyr, p.Trp246Leu or p.Met255Ile) may cause platelet-type von Willebrand disease (24-26). The association between genetic disorders of GP1BA and Bernard-Soulier syndrome (also known as giant platelet syndrome), which is characterized by a low platelet count and giant platelets, has been reported in Iranian, Indian and Kuwaiti populations, even though the gene variants or frameshifts are located in different regions of the GP1BA sequence (27-30). Furthermore, a GP1BA polymorphism has been associated with post-partum hemorrhage (31). In the present study, the same deletion of the GP1BA gene [c.1322_1344del23, p.Ser441TyrfsTer (frameshift and termination), rs770089708] was detected in the proband and the proband's sister. The VWF binding domain is located in the leucine-rich domain (19-281 aa) of the extracellular region. The deletion was located in the extracellular domain of GA1BA. The homology of in this region of the GP1BA protein sequence is low among Homo sapiens (NCBI Reference Sequence: NP_000164), Mus musculus (NCBI Reference Sequence: NP_034456) and Rattus norvegicus (NCBI Reference Sequence: NP_001103124). As the deletion causes termination of GP1BA translation, the heterodimer GP1BA/GP1BB on the platelet surface may be affected. In the ClinVar database, the clinical significance of the GA1BP p.Ser441Tyr fs Ter mutation was rated as 'likely benign', which was according to only one data source. However, this mutation appears to be rare and may still be involved in thrombosis. The mutation most likely leads to the translation of a truncated GP1BA protein, which is soluble, since it cannot be incorporated into the plasma membrane. A detailed functional analysis of this GP1BA mutation requires to be performed in the future.

Results of the whole exome analysis: Unknown rare variants. The whole-exome analysis identified 742, 711 and 750 genes with unknown rare variants in cases II-3, II-1 and III-3, respectively. A Venn diagram presenting shared genes with rare mutations among the three subjects is displayed in Fig. 4. As cases II-3 and II- 1 had presented with venous thrombosis, the 168 identical variants were subjected to a functional annotation analysis via DAVID bioinformatics resources $(14,15)$. The genetic association database disease class for several of these gene mutations is presented in Table III. Among them, 25 genes were involved in human disease and 2 were associated with hematological diseases. The first gene is homeostatic iron regulator (HFE). The function of HFE protein is to regulate iron absorption. Therefore, the defects in HFE result in iron storage disorder and hereditary haemochromatosis (32). Previous studies have indicated an association between thrombosis and hereditary hemochromatosis (with HFE C282Y, H63D and S65C mutations) (33,34). In the present study, cases II- 3 and II- 1 shared a HFE mutation, namely an insertion in a splice region variant at 618-619/1519 of HFE complementary DNA; this mutation has not been recorded in ClinVar and has not been reported by any previous studies, to the best of our knowledge. Although this variant did not alter the coding sequence, this splice site mutation may alter the maturation of HFE mRNA and then result in the production of abnormal proteins. It was hypothesized that this novel HFE mutation may also be a risk factor for venous thrombosis.

The second shared gene associated with hematological diseases was major histocompatibility complex, class I, C (HLA-C). Two missense variants of HLA-C, namely c.98A >C (p.Asp33Ala, rs1071650) and c.121C >A (p.Arg41Ser, rs41555420), were observed in the two subjects via the Clinvar database. The function of these were unknown; however, the Clinvar database did not record them as pathogenic. As HLA-C is highly polymorphic and no published studies have indicated 
Table II. Gene mutations shared between the proband and the proband's sister in ClinVar.

\begin{tabular}{lllr}
\hline Gene symbol & Nucleotide change & Protein change & dbSNP no. \\
\hline SLC4A1 & c.388G $>$ A & p.Gly130Arg & rs121912749 \\
GP1BA & c.1322_1344del23 & p.Ser441TyrfsTer & rs770089708 \\
\hline
\end{tabular}

GP1BA, glycoprotein Ib platelet $\alpha$ subunit; SLC4A1, solute carrier family 4 member 1; dbSNP, single nucleotide polymorphism database.

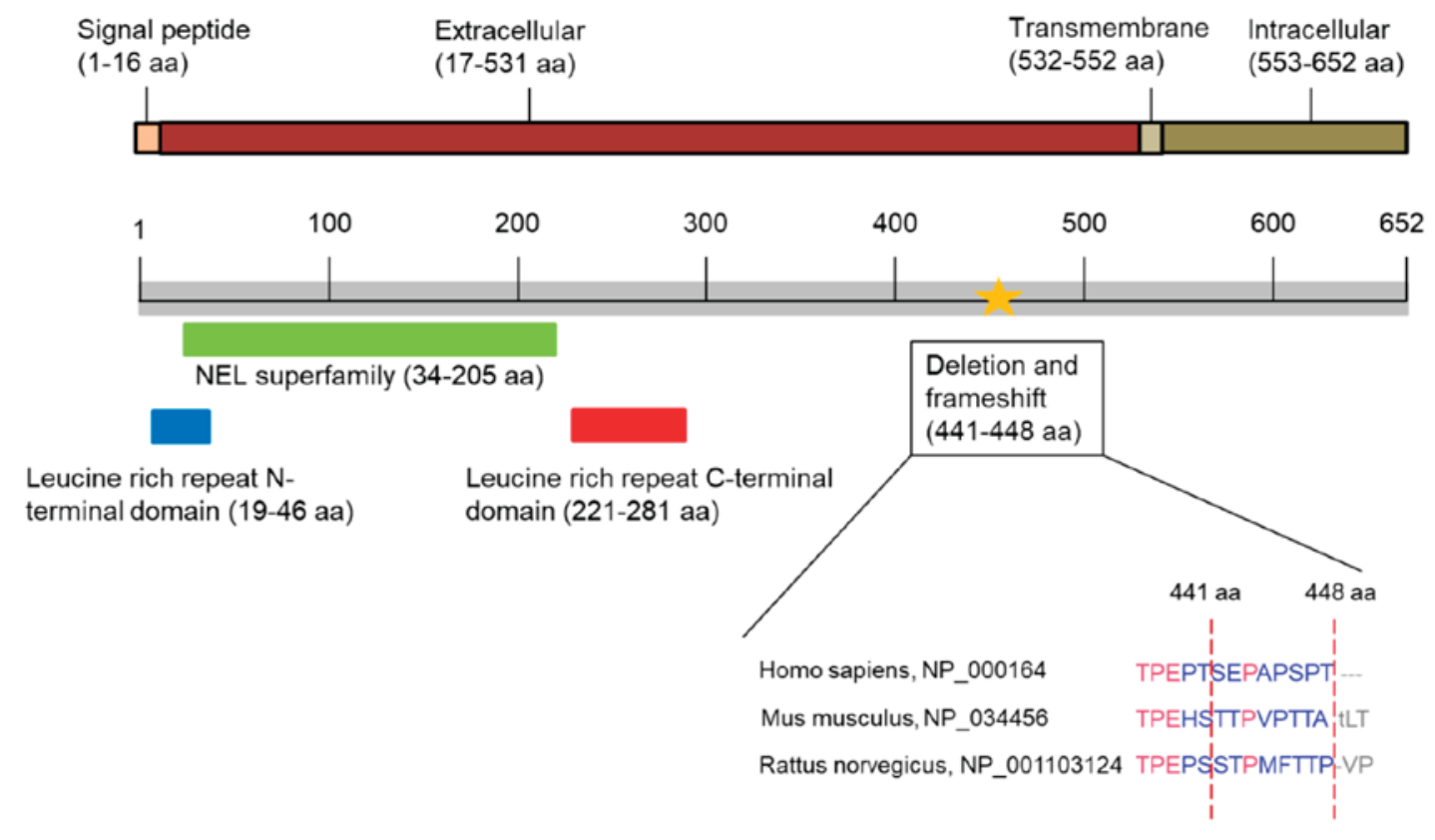

Figure 3. Illustration of protein features and domains of GP1BA. The yellow star indicates the variant of GP1BA identified in the proband and the proband's sister. GP1BA, glycoprotein Ib platelet $\alpha$ subunit.

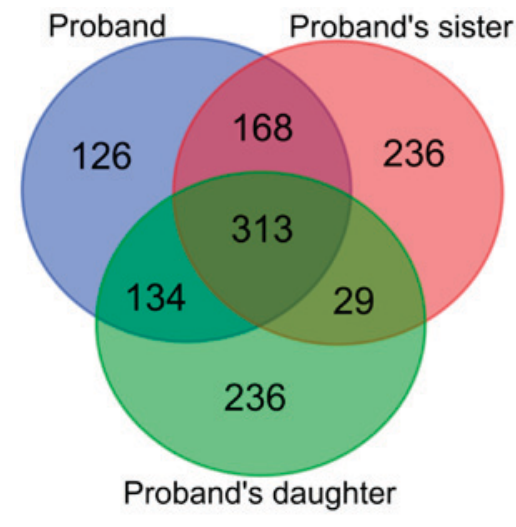

Figure 4. Venn diagram for the genes with identical variants among the proband, the proband's sister and the proband's daughter. A total of 168 identical variants were observed between the proband and proband's sister, and 313 identical variants were observed among the proband, the proband's sister and the proband's daughter.

that these variants of HLA-C are pathogenic, the possibility of HLA-C being involved in abnormal venous thrombosis was ruled out.

Results of whole exome analysis: All rare variants among the proband, proband's sister and proband's daughter. The identical mutations between cases II-3 and II-1 were discussed above, as these two subjects had presented with venous thrombosis, while case III-3 was not considered, as she had not experienced any thrombotic events. However, the possibility of her developing venous thrombosis later in life could not be ruled out. Therefore, the 313 identical variants among cases II-3, II-1 and III-3 were subjected to functional annotation analysis and the genetic association database disease class of certain common mutations is presented in Table IV. Among them, only 5 genes, including HLA-A, HLA-B, major histocompatibility complex, class II, DQ $\beta 1$ (HLA-DQB1), HLA-DRB1 and cytochrome P450 family 2 subfamily D member 6 (CYP2D6), were associated with hematological diseases. HLA-A, HLA-B, HLA-DQB1 and HLA-DRB1 are components of human major histocompatibility complex class I and class II. Human major histocompatibility complex (MHC) is a highly polymorphic gene, which recognises various pathogens. Thus, it was assumed that these gene polymorphisms are not associated with any abnormal venous thrombosis.

CYP2D6 is an important hepatic enzyme involved in the metabolism of 20-25\% of clinical drugs (35). CYP2D6 is highly polymorphic and $>100$ allelic variants of CYP2D6 have been identified (36), several of which do not alter the enzyme function of CYP2D6, although these known CYP2D6 polymorphisms may interfere with 
Table III. Diseases associated with the shared mutations between the proband and the proband's sister.

\begin{tabular}{|c|c|}
\hline Gene symbol & Genetic association database disease class \\
\hline ATF5 & Psych \\
\hline CALY & Psych \\
\hline DOK1 & Cancer \\
\hline EMX2 & Developmental, reproduction \\
\hline FADS1 & Immune, psych \\
\hline GLRB & Neurological \\
\hline $\mathrm{HFE}^{\mathrm{a}}$ & $\begin{array}{l}\text { Aging, cancer, cardiovascular, } \\
\text { hematological, immune, infection, } \\
\text { metabolic, neurological, reproduction, } \\
\text { vision }\end{array}$ \\
\hline HLA-C & $\begin{array}{l}\text { Cancer, cardiovascular, hematological, } \\
\text { immune, infection, metabolic, neurological, } \\
\text { normal variation, pharmacogenomic, } \\
\text { reproduction }\end{array}$ \\
\hline HLA-H & Immune \\
\hline IL2RB & Cancer, immune, infection, psych \\
\hline KRT19 & Unknown (cirrhosis, biliary primary) \\
\hline LTBP4 & Immune \\
\hline MASP1 & Immune \\
\hline MMP10 & Cardiovascular \\
\hline MTF1 & Neurological \\
\hline MADCAM1 & Immune \\
\hline MYO15A & Other \\
\hline NOTCH4 & Immune, neurological, psych \\
\hline PANK2 & Neurological \\
\hline PKHD1 & Renal \\
\hline RBMX & Reproduction \\
\hline RYK & Developmental \\
\hline SCN1B & Neurological \\
\hline SLC4A5 & Cardiovascular \\
\hline ZBTB16 & Cancer \\
\hline
\end{tabular}

andicates the genes associated with hematological diseases. ATF5, activating transcription factor 5; CALY, calcyon neuron specific vesicular protein; DOK1, docking protein 1; EMX2, empty spiracles homeobox 2; FADS1, fatty acid desaturase 1; GLRB, glycine receptor beta; HFE, homeostatic iron regulator; HLA-C, major histocompatibility complex, class I, C; HLA-H, major histocompatibility complex, class I, H (pseudogene); IL2RB, interleukin 2 receptor subunit beta; KRT19, keratin 19; LTBP4, latent transforming growth factor beta binding protein 4; MASP1, mannan binding lectin serine peptidase 1; MMP10, matrix metallopeptidase 10; MTF1, metal regulatory transcription factor 1 ; MADCAM1, mucosal vascular addressin cell adhesion molecule 1; MYO15A, myosin XVA; NOTCH4, NOTCH 4; PANK2, pantothenate kinase 2; PKHD1, PKHD1, fibrocystin/polyductin; RBMX, RNA binding motif protein X-linked; RYK, receptor-like tyrosine kinase; SCN1B, sodium voltage-gated channel beta subunit 1; SLC4A5, solute carrier family 4 member 5; ZBTB16, zinc finger and BTB domain containing 16.

alternative splicing and transcription of the mRNA (37). In addition, CYP2D6 has a frequency of multiplication of $45 \%$ in Asian populations (38). In the present study, a homologous CYP2D6 variant c.1457G >C (p.Ser486Thr, rs1135840) was detected among all three subjects. As the copy number was not determined in the present study, it was not possible to determine whether the subjects carried a CYP2D6 multiplication. According to a previous study, the polymorphisms in CYP2D6*2 with rs1135840 are common in Chinese populations and CYP2D6*2 with rs1135840 retains its normal enzyme function (39). This may imply that the homologous CYP2D6 variant does not affect the enzyme function in this pedigree. Based on the above evidence, the identical gene mutations among the three subjects of the present study may not be major causes of venous thrombosis.

In conclusion, the present study identified common SLC4A1 and GP1BA mutations, which are known in ClinVar, and the common unknown HFE mutation between two female siblings with venous thrombosis (Fig 5). To the best of our knowledge, the present study was the first to report a possible association between venous thrombosis and these mutations in an Asian pedigree.

\section{Acknowledgements}

The authors would like to thank the Center for Research Resources and Development of Kaohsiung Medical University.

\section{Funding}

The present study was supported by grants from the Ministry of Science and Technology (grant nos. MOST 106-2314-B-037-016-MY2,MOST 106-2320-B-037-029-MY3 and MOST 107-2320-B-037-011-MY3), Kaohsiung Medical University Hospital (grant nos. KMUHS10701, KMUHS10712, KMUH106-6R15 and KMUH106-6R77), and the Kaohsiung Medical University (grant no. KMU-DK108003).

\section{Availability of data and materials}

The analyzed data sets generated during the present study are available from the corresponding author on reasonable request.

\section{Authors' contributions}

WC, MY and PK designed the study; WC and CS enrolled the patients; WC, KL, JS, MY and PK analyzed the data and interpreted the results; and $\mathrm{WC}$, and MY wrote the manuscript. The final version of the manuscript has been read and approved by all authors, and each author believes that the manuscript represents honest work.

\section{Ethical approval and consent to participate}

The present study was approved by the Institutional Review Board of KMUH (Kaohsiung, Taiwan). All subjects provided written informed consent.

\section{Patient consent for publication}

Not applicable. 
Table IV. Diseases associated with the mutations shared between the proband, the proband's sister and the proband's daughter.

\section{Gene symbol}

\section{PRDM2}

ALDH5A1

APOL4

ATR

ATXN1

ATXN3

ATN1

CACNA1A

CCL3L3

CX3CR1

CYP2C19

CYP2D6 $^{\mathrm{a}}$

DACH2

DOCK 3

FAAH

FGFR1

FLG

FMO2

GPX1

GNPAT

GDF5

GHRHR

IFI27

KRT18

KIR3DL2

KIR3DL3

KIR2DL1

LILRB1

LILRB4

HLA-A $^{\mathrm{a}}$

HLA-B $^{\mathrm{a}}$

HLA-DQA1

HLA-DQB $1^{\text {a }}$

HLA-DRB $1^{\text {a }}$

HLA-DRB5

MAP3K1

MUC2

MUC3A

MUC4

MUC5AC

MUC6

MSH6

MYOC

NIPA1
Genetic association database disease class

\section{Cancer}

Neurological, psych

Psych,

Cancer, cardiovascular

Cardiovascular, neurological, psych

Neurological, psych

Cardiovascular, neurological, psych

Neurological, psych

Immune, infection

Cancer, cardiovascular, immune, infection, renal, vision

Cancer, cardiovascular, immune, infection, metabolic, neurological, normal variation, pharmacogenomic, psych, reproduction

Aging, cancer, cardiovascular, chemdependency, hematological, immune, infection, metabolic, neuro-logical, normal variation, pharmacogenomic, psych, renal, reproduction, vision

Reproduction

Psych

Chemdependency, metabolic, psych

Developmental

Immune

Cancer

Cancer, cardiovascular, immune, metabolic, pharmacogenomic, psych, renal, vision

Psych

Metabolic

Cancer, developmental, metabolic

Infection

Other

Cancer, immune, normal variation

Cancer, normal variation

Cancer, cardiovascular, immune, infection, normal variation, renal

Immune

Immune

Aging, cancer, cardiovascular, hematological, immune, infection, metabolic, neurological, normal variation, pharmacogenomic, psych, reproduction

Cancer, cardiovascular, hematological, immune, infection, metabolic, neurological, normal variation, pharmacogenomic, reproduction

Cancer, cardiovascular, developmental, immune, infection, metabolic, neuro logical, normal variation, pharmacogenomic, psych, renal, reproduction

Cancer, cardiovascular, hematological, immune, infection, metabolic, neuro logical, normal variation, pharmacogenomic, psych, renal, reproduction, vision

Cancer, cardiovascular, hematological, immune, infection, metabolic, neuro logical, normal variation, pharmacogenomic, psych, renal, reproduction, vision

Cancer, cardiovascular, immune, infection, metabolic, neurological

Cancer

Immune

Immune

Immune, reproduction

Immune

Infection

Cancer

Vision

Immune 
Table IV. Continued.

Gene symbol

Genetic association database disease class

\section{PER3}

RAI1

SLC5A4

SYN2

TNS1

TCN2

TTN
Aging, chemdependency, neurological, psych

Smith-magenis syndrome

Psych

Psych

Developmental

Cancer, cardiovascular, developmental, immune, infection, metabolic, neuro logical, reproduction Cardiomyopathy dilated $1 \mathrm{G}$, cardiomyopathy familial hypertrophic, 9 , muscular dystrophy

limb-girdle type 2J, myopathy early-onset with fatal cardiomyopathy, myopathy, proximal with early respiratory muscle involvement, tibial muscular dystrophy tardive

Immune, renal

XYLT2

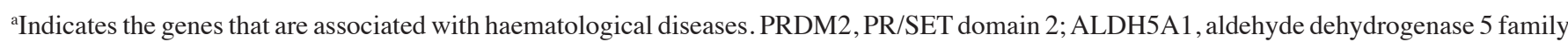
member A1; APOL4, apolipoprotein L4; ATR, ATR serine/threonine kinase; SERPINA2, serpin family A member 2 (gene/pseudogene); ANTXR1, ANTXR cell adhesion molecule 1; ATXN1, ataxin 1; ATXN3, ataxin 3; ATN1 atrophin 1, CACNA1A, calcium voltage-gated channel subunit alpha1 A; CCL3L3, C-C motif chemokine ligand 3 like 3; CX3CR1, C-X3-C motif chemokine receptor 1; CYP2C19, cytochrome P450 family 2 subfamily C member 19; CYP2D6, cytochrome P450 family 2 subfamily D member 6; DACH2, dachshund family transcription factor 2; DOCK3, dedicator of cytokinesis 3; FAAH, fatty acid amide hydrolase; FA2H, fatty acid 2-hydroxylase; FGFR1, fibroblast growth factor receptor 1; FLG, filaggrin; FGFR1, fibroblast growth factor receptor 1; FMO2, flavin containing monooxygenase 2; FMO4, flavin containing monooxygenase 4; GPX1, glutathione peroxidase 1; GNPAT, glyceronephosphate O-acyltransferase; GDF5, growth differentiation factor 5; GHRHR, growth hormone releasing hormone receptor; IFI27, interferon alpha inducible protein 27; KRT18, keratin 18; KIR3DL2, killer cell immunoglobulin like receptor, three Ig domains and long cytoplasmic tail 2; KIR3DL3, killer cell immunoglobulin like receptor, three Ig domains and long cytoplasmic tail 3; KIR2DL1, killer cell immunoglobulin like receptor, two Ig domains and long cytoplasmic tail 1; LILRB1, leukocyte immunoglobulin like receptor B1; LILRB4, leukocyte immunoglobulin like receptor B4; HLA-A, major histocompatibility complex, class I, A; HLA-B, major histocompatibility complex, class I, B; HLA-DQA1, major histocompatibility complex, class II, DQ alpha 1; HLA-DQB1, major histocompatibility complex, class II, DQ beta 1; HLA-DRB1, major histocompatibility complex, class II, DR beta 1; HLA-DRB5, major histocompatibility complex, class II, DR beta 5; MAP3K1, mitogen-activated protein kinase kinase kinase 1; MUC2, mucin 2, oligomeric mucus/gel-forming; MUC3A, mucin 3A, cell surface associated; MUC4, mucin 4, cell surface associated; MUC5AC, mucin 5AC, oligomeric mucus/gel-forming; MUC6, mucin 6, oligomeric mucus/gel-forming; MSH6, mutS homolog 6; MYOC, myocilin; NIPA1, NIPA magnesium transporter 1; PER3, period circadian regulator 3; RAI1, retinoic acid induced 1; RNASE12, ribonuclease A family member 12 (inactive); SLC5A4, solute carrier family 5 member 4; SYN2, synapsin II; TNS1, tensin 1; TCN2, transcobalamin 2; TTN, titin; XYLT2, xylosyltransferase 2.

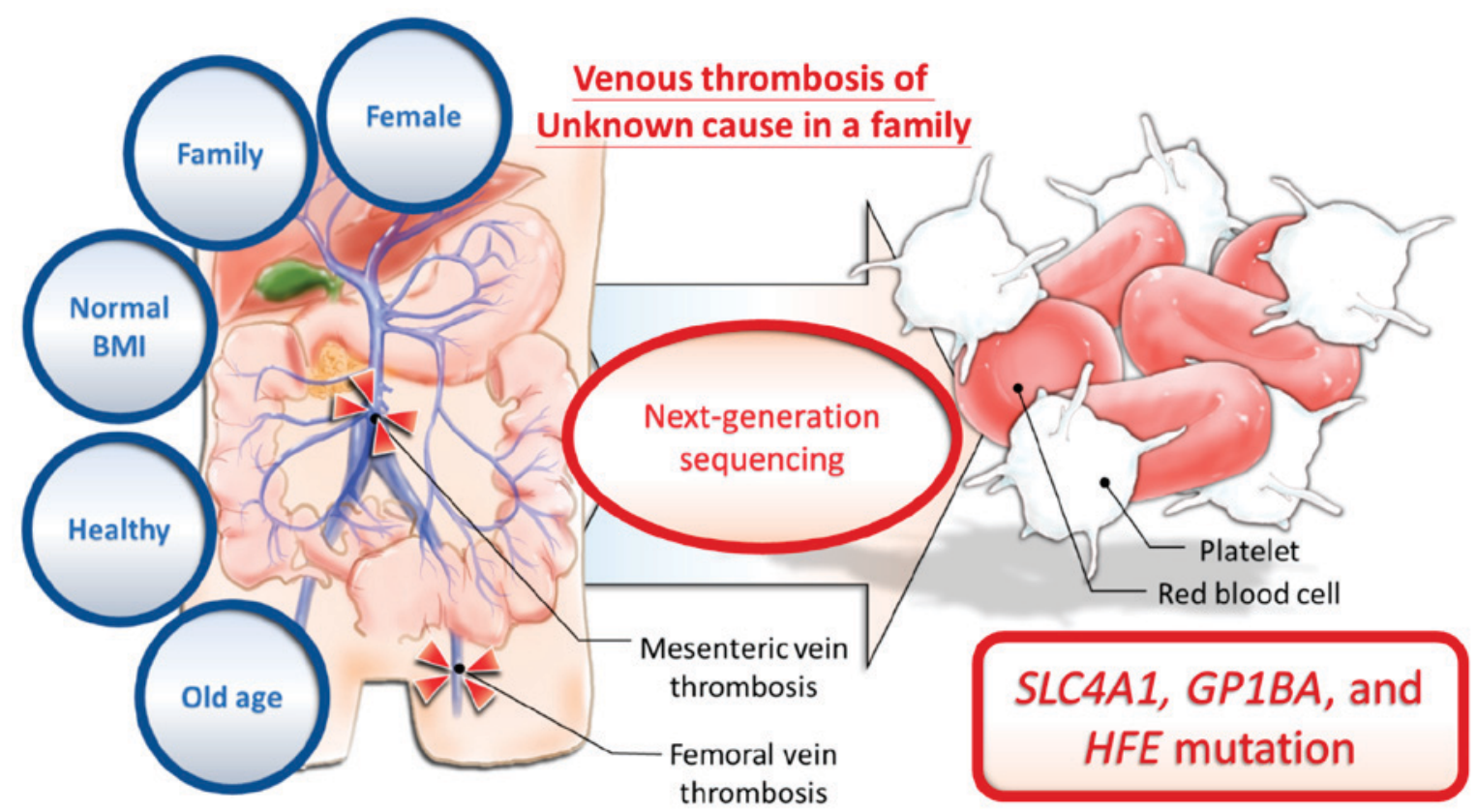

Figure 5. Summary scheme. In the present study, mutations in GP1BA, SLC4A1 and HFE were identified in a female patient and two of her pedigree members with venous thrombosis of unknown cause via next-generation sequencing. GPIBA is associated with diseases of platelets, and SCL4A1 and HFE are associated with diseases involving red blood cells. BMI, body mass index; GP1BA, glycoprotein Ib platelet $\alpha$ subunit; SLC4A1, solute carrier family 4 member 1 ; HFE, homeostatic iron regulator. 


\section{Competing interests}

The authors declare that they have no competing interests.

\section{References}

1. Rosendaal FR and Reitsma PH: Genetics of venous thrombosis. J Thromb Haemost 7 (Suppl 1): S301-S304, 2009.

2. Miyata T, Maruyama K, Banno F and Neki R: Thrombophilia in East Asian countries: Are there any genetic differences in these countries? Thromb J 14 (Suppl 1): S25, 2016.

3. Makris M, Rosendaal FR and Preston FE: Familial thrombophilia: Genetic risk factors and management. J Intern Med Suppl 740: 9-15, 1997.

4. Lotta LA, Wang M, Yu J, Martinelli I, Yu F, Passamonti SM, Consonni D, Pappalardo E, Menegatti M, Scherer SE, et al: Identification of genetic risk variants for deep vein thrombosis by multiplexed next-generation sequencing of 186 hemostatic/pro-inflammatory genes. BMC Med Genomics 5: 7, 2012.

5. Lotta LA, Tuana G, Yu J, Martinelli I, Wang M, Yu F, Passamonti SM, Pappalardo E, Valsecchi C, Scherer SE, et al: Next-generation sequencing study finds an excess of rare, coding single-nucleotide variants of ADAMTS13 in patients with deep vein thrombosis. J Thromb Haemost 11: 1228-1239, 2013.

6. Morange PE, Suchon P and Trégouët DA: Genetics of venous thrombosis: Update in 2015. Thromb Haemost 114: 910-919, 2015.

7. Sulger E and Gonzalez L: Mesenteric venous thrombosis. StatPearls [Internet]. Treasure Island (FL): StatPearls Publishing, 2017. https://www.ncbi.nlm.nih.gov/books/NBK459184/ [Accessed on January 15, 2018]

8. Singh M, Long B and Koyfman A: Mesenteric ischemia: A deadly miss. Emerg Med Clin North Am 35: 879-888, 2017.

9. Bolger AM, Lohse M and Usadel B: Trimmomatic: A flexible trimmer for Illumina sequence data. Bioinformatics 30: 2114-2120, 2014.

10. Li H and Durbin R: Fast and accurate short read alignment with Burrows-Wheeler transform. Bioinformatics 25: 1754-1760, 2009.

11. DePristo MA, Banks E, Poplin R, Garimella KV, Maguire JR, Hartl C, Philippakis AA, del Angel G, Rivas MA, Hanna M, et al: A framework for variation discovery and genotyping using next-generation DNA sequencing data. Nat Genet 43: 491-498, 2011.

12. McLaren W, Gil L, Hunt SE, Riat HS, Ritchie GR, Thormann A, Flicek $\mathrm{P}$ and Cunningham F: The ensembl variant effect predictor. Genome Biol 17: 122, 2016.

13. Landrum MJ, Lee JM, Benson M, Brown G, Chao C, Chitipiralla S, Gu B, Hart J, Hoffman D, Hoover J, et al: ClinVar: Public archive of interpretations of clinically relevant variants. Nucleic Acids Res 44: D862-D868, 2016.

14. Chen CH, Yang JH, Chiang CWK, Hsiung CN, Wu PE, Chang LC, Chu HW, Chang J, Song IW, Yang SL, et al: Population structure of Han Chinese in the modern Taiwanese population based on 10,000 participants in the Taiwan Biobank project. Hum Mol Genet 25: 5321-5331, 2016

15. Huang da W, Sherman BT and Lempicki RA: Systematic and integrative analysis of large gene lists using DAVID bioinformatics resources. Nat Protoc 4: 44-57, 2009.

16. Huang da W, Sherman BT and Lempicki RA: Bioinformatics enrichment tools: Paths toward the comprehensive functional analysis of large gene lists. Nucleic Acids Res 37: 1-13, 2009.

17. Kunicki TJ, Williams SA and Nugent DJ: Genetic variants that affect platelet function. Curr Opin Hematol 19: 371-379, 2012.

18. Korte W, Poon MC, Iorio A and Makris M: Thrombosis in inherited fibrinogen disorders. Transfus Med Hemother 44: 70-76, 2017

19. Liu Y, Yang J and Chen LM: Structure and function of SLC4 family [formula: See text] transporters. Front Physiol 6: 355, 2015.

20. Perkins LA, Jones SF and Bhargava RS: Dural venous thrombosis following splenectomy in a patient with hereditary spherocytosis. South Med J 102: 542-545, 2009.
21. Agarwal SK, Binbrek AS, Thompson JA and Siddiqui SA: Massive pulmonary embolism and acute limb ischaemia in a patient of hereditary spherocytosis and patent foramen ovale. Heart Lung Circ 19: 742-744, 2010

22. Ozaki Y, Asazuma N, Suzuki-Inoue K and Berndt MC: Platelet GPIb-IX-V-dependent signaling. J Thromb Haemost 3: 1745-1751, 2005

23. Watkins NA, Gusnanto A, de Bono B, De S, Miranda-Saavedra D, Hardie DL, Angenent WG, Attwood AP, Ellis PD, Erber W, et al: A HaemAtlas: Characterizing gene expression in differentiated human blood cells. Blood 113: e1-e9, 2009.

24. Enayat S, Ravanbod S, Rassoulzadegan M, Jazebi M, Tarighat S, Ala F, Emsley J and Othman M: A novel D235Y mutation in the GP1BA gene enhances platelet interaction with von Willebrand factor in an Iranian family with platelet-type von Willebrand disease. Thromb Haemost 108: 946-954, 2012.

25. Lavenu-Bombled C, Guitton C, Dupuis A, Baas MJ,Desconclois C, Dreyfus M, Li R, Caron C, Gachet C, Fressinaud E and Lanza F: A novel platelet-type von Willebrand disease mutation (GP1BA p.Met255Ile) associated with type 2B 'Malmö/New York' von Willebrand disease. Thromb Haemost 116: 1070-1078, 2016.

26. Woods AI, Sanchez-Luceros A, Bermejo E, Paiva J, Alberto MF, Grosso SH, Kempfer AC and Lazzari MA: Identification of p.W246L as a novel mutation in the GP1BA gene responsible for platelet-type von Willebrand disease. Semin Thromb Hemost 40: 151-160, 2014.

27. Ali S, Ghosh K and Shetty S: Novel genetic abnormalities in bernard-soulier syndrome in india. Ann Hematol 93: 381-384, 2014.

28. Shlebak A, Poles A, Manning R, Almuhareb S, De La Funte J, Mitchell M and Lucas G: A novel homozygous c.800C $>$ G substitution in GP1BA exon 2 in a kuwaiti family with bernard-soulier syndrome. Acta Haematol 134: 193-198, 2015.

29. Afrasiabi A, Lecchi A, Artoni A, Karimi M, Ashouri E, Peyvandi F and Mannucci PM: Genetic characterization of patients with bernard-soulier syndrome and their relatives from Southern Iran. Platelets 18: 409-413, 2007.

30. Ali S, Shetty S and Ghosh K: A novel mutation in GP1BA gene leads to mono-allelic bernard soulier syndrome form of macrothrombocytopenia. Blood Coagul Fibrinolysis 28: 94-95, 2017.

31. Biguzzi E, Franchi F, Acaia B, Ossola W, Nava U, Paraboschi EM, Asselta R and Peyvandi F: Genetic background and risk of postpartum haemorrhage: Results from an Italian cohort of 3219 women. Haemophilia 20: e377-e383, 2014.

32. Valenti L and Pelusi S: HFE mutations and iron in hemodialysis patients. Hemodial Int 21 (Suppl 1): S47-S57, 2017.

33. Brown K, Luddington R, Taylor SA, Lillicrap DP and Baglin TP Risk of venous thromboembolism associated with the common hereditary haemochromatosis Hfe gene (C282Y) mutation. Br J Haematol 105: 95-97, 1999.

34. Dionisio Tavares Niewiadonski V, Dos Santos Bianchi JV, de Almeida-Neto C, Gaburo N Jr and Sabino EC: Evaluation of a high throughput method for the detection of mutations associated with thrombosis and hereditary hemochromatosis in Brazilian blood donors. PLoS One 10: e0125460, 2015

35. Ingelman-Sundberg M: Pharmacogenetics of cytochrome P450 and its applications in drug therapy: The past, present and future. Trends Pharmacol Sci 25: 193-200, 2004.

36. Wang B, Yang LP, Zhang XZ, Huang SQ, Bartlam M and Zhou SF: New insights into the structural characteristics and functional relevance of the human cytochrome P450 2D6 enzyme. Drug Metab Rev 41: 573-643, 2009.

37. Wang D, Poi MJ, Sun X, Gaedigk A, Leeder JS and Sadee W: Common CYP2D6 polymorphisms affecting alternative splicing and transcription: Long-range haplotypes with two regulatory variants modulate CYP2D6 activity. Hum Mol Genet 23: 268-278, 2014

38. Sistonen J, Fuselli S, Levo A and Sajantila A: CYP2D6 genotyping by a multiplex primer extension reaction. Clin Chem 51: 1291-1295, 2005

39. Goh LL, Lim CW, Sim WC, Toh LX and Leong KP: Analysis of genetic variation in CYP450 genes for clinical implementation. PLoS One 12: e0169233, 2017. 